\title{
Research on Product Natural User Interface Design based on User Experience
}

\author{
Chen, Leyang ${ }^{1, a}$, Wu Jian ${ }^{2, b}$ and Hu Minghui ${ }^{3, c}$ \\ ${ }^{1}$ Beijing Institute of Graphic Communication, Beijing 102600, China \\ ${ }^{2}$ Beijing Institute of Graphic Communication, Beijing 102600, China \\ ${ }^{3}$ Beijing Institute of Graphic Communication, Beijing 102600, China

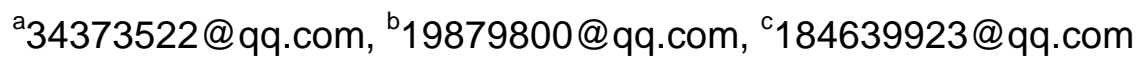

Keywords: User Experience, Natural User Interface Design

Abstract. Interface is the information platform for Man-Computer Exchange. It's the key factor of user experience. This thesis concludes the principle and methods of product interface design, in the view of user experience, by research on interface design of industrial products. It uses NEST learning temperature controller as example, by research on target users, to construct user model and realize isomorphism of product interface design.

\section{Introduction}

With the development of technology, human society has enter a digital era marks by information. Nowadays products design has changed from basic function pursuit to user experience center. Users are more concentrate on user's mental reaction in spiritual plane. Designers are no longer design products, but design a new way of life. In such circumstance, it's necessary for nowadays designer to reflect on principle and method of traditional product interface design, and fully understand new interface design concept in new experience era.

\section{Users Experience and Product Interface Design}

\section{User Experience}

User Experience is the mental feeling constructed in the process when users using products or enjoying services. It involves all aspects of interaction process of man and products ${ }^{[1]}$, including the service provided by products [1]. Bill Gates, who has studied user interface and firstly raise natural user interface theory. Though the function and manipulate method are totally the same, if interface can learn and adapt people's application habit, the products design will have great progress.

A good design of users experience is a long, circulating process, as shown in Fig. 1, while the unpleasant design is one-time straightforward. Designers are always expect users experience can become a beneficial cycle, and make users use products again, at last forming loyalty to the products.

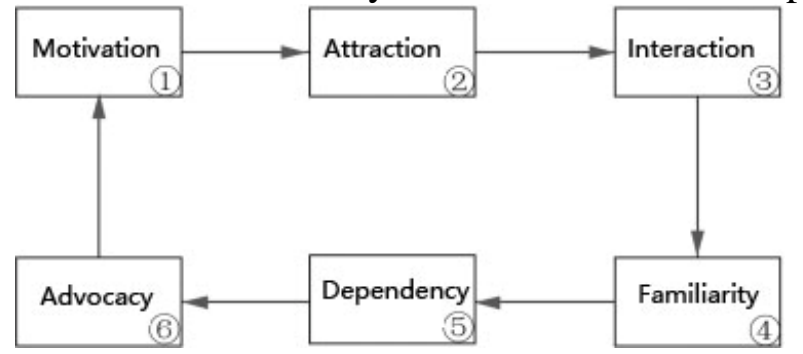

Fig 1. Life Cycle Model of User Experience

User Motivation is the first step of User Experience Life Cycle, about what purpose users want to achieve by the products. Second step is what kind of product can attract customers. The third step is how it feels to interact with products, whether it is easy to use and whether it can create pleasing experience. The forth step is whether customer willing to continue to use the product and whether gradually familiar with products and its service. The fifth step is whether the customers get used to use 
the products and whether having dependency on the product. The sixth step is whether will it create advocacy and recommend it to their friends.

\section{Relation between User Experience and Products Natural User Interface Design}

Interface is a platform of information communication. Products interface design, including hard interface and soft interface, should achieve isomorphism, which means transmit information between users and designers accurately and rapidly. That means design information should be accurately understood by users. It should also create mental communication in aesthetic consciousness and product function between users and designers. So the products, as carrier of period economics, scientific and humanity spirits, should more concentrate on user experience, to make itself more simple, easy to use and create pleasing and funny experience.

The natural user interface design, which is based on user experience, is user research centered. From the aspect of products users, design object is not only the product itself, but also the whole process of user experience, throughout the whole life cycle of product ${ }^{[2]}$. Designer should pay more attention to focus on users, study users, understand and respect different culture and influence products experience quality by design process. They should also make products interface more learning type and intelligent type, to be able to learn and adapt users' custom.

\section{Problems of Traditional Products Interface Design}

The process when people use products is the process they interact with products. The process always accompanied by information isomorphism. High tech brought us to colorful material world, but what's coming with it? Perhaps it's pleasing experience, perhaps unexpected embarrassment. Application of high tech makes products more powerful, at the same time more complicated to understand and use. Users usually experience frustration and tension, rather than pleasing experience.

(1) Because execution of products function no longer uses only traditional sensible method, but adds more invisible electrical information operation. It makes it difficult to explain products interior function and application based on its appearance.

(2) Software interface is mostly exclusively designed by software engineer. Though it realizes software's function, but it lacks of user friendly, which means understanding, aesthetic, fault tolerance, learning.

(3) Product interface design researchers are mostly researching on mechanism or human physiology angle rather than user experience angle.

\section{Product Interface Design Principle based on User Experience}

The key of product interface design based on user experience is user centered, to build a bridge between designer and users, to involve users in product design and test process and get feedback from them. And then continuously improve products based on users' requirement and feedback, until producing satisfying product. Relation Model is shown in Fig 2.

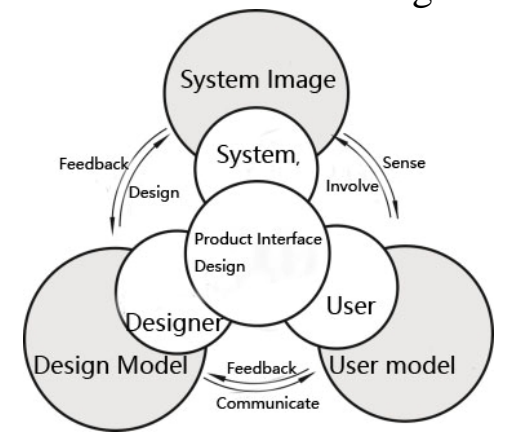

Fig 2. Product Interface Design Model 


\section{Construct Users Model}

Take Learning Temperature Controller as example, we will illustrate research and effects of users thought model, task model, semantics model, and irrational paradigm.

\section{Construct User Thought Model}

Thought Model is the method that user's brain simulates knowledge. In order to increase ease of use, designer should adapt to user's thought model, to connect products with users manipulate behavior. The user thought model, take NEST learn temperature controller as example, includes: (1) Study user's relation with other entities, including users, family and temperature controller, from user manipulate angle rather than designer angle ${ }^{[3]}$. (2) Users manipulate knowledge and other necessary knowledge about NEST temperature controller is the foundation of their learning and using the controller. The interface design should minimize the quantity and difficulty of those knowledge. (3) Composition of user action, including sensing, thought, will, physical action, and emotion. The design of controller should create and comply action condition needed by users.

Constructing User thought model can clearly understand users understanding, thought and learning capacity, and guide interface design. Since user's understanding, thought and learning capacity are limited, NEST temperature controller interface design should minimize difficulty of using. The interface should be as simple as possible and use multi-channel user interface.

\section{Construct User Task Model}

Task model are also called manipulate process model, which means the purposed action process when users finish all kinds of tasks. [3] When users facing task, their rational manipulation is always following certain method, instruction and steps. Take NEST temperature controller as example, the model research generally includes: (1) User's motivation and purpose of using. Where is the interest and expectation point of the function and service of NEST temperature controller? How to break down a complicated target to several simple sub-targets? (2) User's action plan. To accomplish purpose like temperature control and inquiry, when and where do they use temperature controller? How to manipulate? How do they think and choose manipulate process? (3) User's actual manipulate process. How do they start to manipulate? How to install? (4) Task Evaluation. After user finished tasks like temperature inquiry and switch on/off of air blower, they should exam and evaluate the result of NEST temperature controller. How do they evaluate? What's the result? What kind of difficulty they met when using NEST temperature controller? What methods can correct mistakes?

By construct NEST temperature controller user task model, we can understand the main purpose of user is to inquire temperature, switch on/off and whether do they hope to realize remote control of the controller. Normal process of manipulation on the controller is: Temperature inquiry, Insert comfort temperature, Enter function selection, Switch on/off.

\section{Construct User Semantics Model}

Product Semantic believes design shouldn't be mechanism function origin, but should be human origin. Products should be able to "speak" for themself, to tell users what kinds of people do they fit, what's the function, how to manipulate. So in product interface design ${ }^{[4]}$, designer should be equipped with knowledge about users, to make product understood on sight and not require lots of energy to learn again. NEST temperature controller as example, the Semantics model generally includes: (1) Users experience knowledge of manipulating NEST temperature controller; (2) Ordinary words they use to manipulate NEST temperature controller; (3) Logical thought formed by user development about NEST temperature controller. This words action content construct user Semantics Model and create baseline for designer. It provides manipulate condition for NEST temperature controller interface and accurately express its meaning. 


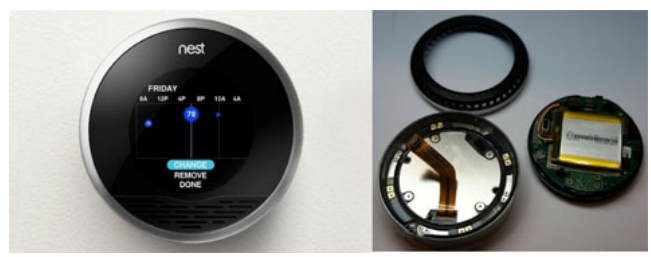

Fig 4. (Left) Interface of NEST Temperature Controller Fig 5. (Right) Interior Figure of NEST Temperature Controller

Interface and interior of NEST Temperature Controller are shown in Fig 4 and Fig 5. We can see that the interface design of the controller is very simple. The interface design's words are very clear and take full consideration about customer's function requirement and visibility.

\section{Construct Irrational User Model}

Human ration has certain limit. It will become irrational when the limit is exceeded. Condition so called "Irrational" means that human action is no longer constrained by purpose, motive and behavior regulation. Such Irrational is shown in all aspects, including sense, behavior, thought, will and motion, and user operating mistakes. For example, human's sense, like vision, auditory, tactual sensation etc. are irrational. User's thought are mostly irrational. Users usually have their own habit to operating NEST Temperature Controller, rather than following certain standard operation. Users forget things easily. Users might misunderstand service item on NEST Temperature Controller interface. User can abnormally operate NEST Temperature Controller interface. As a result, NEST Temperature Controller interface should have certain fault tolerant, which can detect mistake and provide simple, intelligibly problem solving method.

Constructing irrational user model provide guiding for designing various warning interface of NEST temperature controller and make it more intelligent.

\section{Conclusions}

User experience guilds products and service development, and it had become decisive factor of design. In product interface design, designers and users should build up communication mediums, go deep into user research, builds up reasonable user thought model, user task model and semantics model, and pay attention to user irrational paradigm. The target is to quicker transmit design information of product interface between designers and users, to let customer accurately understand and have pleasing and funny experience and to form beneficial cycle in users experience life cycle.

\section{Acknowledgements}

This work was financially supported by the Institute Level Projects Funded by KM201310015009 ,

Beijing Municipal Education Commission tech surface project.

\section{References}

[1] Shijian Luo, Shangshang Zhu: User Experience and Products Creative Design [M]. (China Machine Press Beijing 2010)

[2] Kunqian Wang, Jiehui Lin, Renping Xu in: Information Isomorphism in Product Interface Design [J]. Packaging Engineering 2006 , 27 ( 3 ) : 151-152

[3] Leshan Li: Basic Thought of Industrial Design [M] (China Architecture and Building Press, Beijing 2007 ) 
[4] Bo Ouyang, Yun He in: User Research and User Experience Design [J]. Journal of Jiangsu University(2006) 27 ( 5A ) : 55-57 